\title{
Desgastes dentários em praticantes de exercícios físicos de musculação
}

Dental wear among practicioners of bodybuilding physical exercises

Desgaste dental en practicantes de musculación

Mariana Silva de Bessa ${ }^{1}$

Erik Vinícius Martins Jácome ${ }^{1}$

Marquiony Marques dos Santos ${ }^{2}$

Daniela Mendes da Veiga Pessoa

Gilmara Celli Maia de Almeida $^{1}$

Jamile Marinho Bezerra de Oliveira Moura ${ }^{1}$

${ }^{1}$ Departamento de Odontologia, Universidade do Estado do Rio Grande do Norte (UERN), 59.300-000, Caicó-RN, Brasil

${ }^{2}$ Departamento de Enfermagem, Universidade do Estado do Rio Grande do Norte (UERN), 59.300-000, Caicó-RN, Brasil

\section{Resumo}

Os desgastes dentários conhecidos como atrição, abfração, erosão e abrasão, são considerados alterações fisiológicas ou patológicas, levando em consideração seus fatores etiológicos. Desta forma, o presente estudo objetivou avaliar a frequência de desgastes dentários em praticantes de exercícios físicos de musculação do município de Caicó, Rio Grande do Norte e o conhecimento dos mesmos sobre estes desgastes. A amostra foi constituída por 260 praticantes de musculação de 5 academias do município e para a coleta dos dados utilizou-se questionários e exame clínico intraoral. Foi realizada análise descritiva dos dados e para avaliar a relação entre os desgastes dentários e as variáveis independentes foram utilizados os testes estatísticos: t de Student, Qui-quadrado de Pearson e Correlação de Pearson, considerando um nível de significância de $5 \%$. A presença de pelo menos um tipo de desgaste foi observada em $78,9 \%$ dos praticantes de musculação. Destes, $65,8 \%$ tinham apenas um tipo de desgaste, $12,3 \%$ dois tipos e $0,8 \%$ três tipos. Dos desgastes dentários estudados, a atrição e a abfração foram os mais frequentes, sendo $80,9 \%$ e $15,8 \%$ respectivamente. Dos praticantes, $91,9 \%$ não tinham conhecimento sobre os desgastes dentários. Concluiu-se que com o avanço da idade, maior a frequência de atrição e abfração. A abfração foi mais frequente naqueles que praticavam musculação até 4 vezes na semana. A atrição e a abfração foram as lesões dentárias de desgaste mais desconhecidas, podendo implicar em deficiências na prevenção dessas lesões.

Descritores: Anormalidades Dentárias; Comportamento Alimentar; Desgaste dos Dentes; Treinamento de Resistência.

\section{Abstract}

Dental wear known as attrition, abfraction, erosion and abrasion are considered physiological or pathological changes, taking into account their etiological factors. Thus, the present study aimed to assess the frequency of dental wear in practitioners of bodybuilding physical exercises in the municipality of Caicó, Rio Grande do Norte and their knowledge about these wear and tear. The sample consisted of 260 bodybuilders from 5 gyms in the Caicó city. Questionnaires and intraoral clinical examination were used to collect the data. Data Descriptive analysis was performed and Student's t, Pearson's chi-square and Pearson's correlation tests were used to evaluate the relationship between dental wear and the independent variables, considering a significance level of $5 \%$. At least one type of dental wear alteration was observed in $78.9 \%$ of bodybuilders from whom, $65.8 \%$ had only one type of wear, $12.3 \%$ had two types and $0.8 \%$ had three types. Of the dental wear studied, attrition and abfraction were the most frequent, which are $80.9 \%$ and $15.8 \%$ respectively. Concerning bodybuilding practitioners, $91.9 \%$ were unaware of dental wear. It was concluded that as the age increases, the frequency of attrition and abfraction was higher as well. The abfraction was more frequent among bodybuilders that practiced bodybuilding up to 4 times a week. The attrition and the abfraction were the most unknown injuries, which may result in deficiencies in the prevention of dental wear changes.

Descriptors: Dental Abnormalities; Food Behavior; Tooth Wear; Resistance Training.

\section{Resumen}

Las lesiones de desgaste dental conocidas como atrición, abfración, erosión y abrasión son consideradas cambios fisiológicos o patológicos, teniendo en cuenta sus factores etiológicos. El presente estudio tuvo como objetivo evaluar la frecuencia de las lesiones de desgaste dental en los practicantes de entrenamiento de resistencia en el municipio de Caicó, Río Grande del Norte y su conocimiento sobre estas lesiones. La muestra consistió en 260 fisicoculturistas de 5 gimnasios de la ciudad y se utilizaron cuestionarios y exámenes clínicos intraorales para recolectar los datos. Se realizó un análisis descriptivo de los datos y para evaluar la relación entre las lesiones de desgaste dental y las variables independientes, se utilizaron pruebas estadísticas: $t$ de Student, Chi-cuadrado de Pearson y correlación de Pearson, considerando un nivel de significancia del $5 \%$. Se observó la presencia de al menos un tipo de desgaste en el 78,9\% de los practicantes. De estos, el $65.8 \%$ tenía solo un tipo de desgaste, el $12.3 \%$ dos tipos y el $0.8 \%$ tres tipos. La atrición y la abfracción fueron las lesiones más frecuentes, con $80.9 \%$ y $15.8 \%$ respectivamente. Entre los practicantes, el $91.9 \%$ desconocía las lesiones de desgaste. Se concluyó que con el aumento de la edad, aumenta la frecuencia de la atrición y de la abfracción. La abfracción fue más frecuente en aquellos que practicaban fisicoculturismo hasta 4 veces por semana. La atrición y la abfracción fueron las lesiones de desgaste dental más desconocidas, lo que puede conducir a la deficiencia en la prevención de estas lesiones.

Descriptores: Anomalías Dentarias; Conducta Alimentaria; Desgaste de los Dientes; Entrenamiento de Resistencia.

Os desgastes dentários, tais como atrição, abfração, erosão e abrasão, também conhecidos como perda da superfície dos dentes ou lesões não cariosas, são considerados como um processo fisiológico que ocorre com o decorrer do envelhecimento, mas podem ser patológicos quando o grau de destruição traz problemas funcionais, estéticos ou de sensibilidade dentária ${ }^{1}$.

A atrição dental consiste no desgaste mecânico das estruturas dentárias causada pelo contato entre dentes antagonistas durante a oclusão. A abfração é a perda de estrutura dental por repetida pressão (trauma oclusal) sobre os dentes mediante um estresse oclusal. Por sua vez, a erosão é definida como sendo a perda de estrutura dental em decorrência de substâncias químicas e não envolve a ação dos produtos bacterianos, enquanto a abrasão dental está relacionada com a perda de substância calcificada devido a algum processo mecânico anormal ${ }^{2}$. 
Atualmente, a prática de exercícios físicos vem sendo cada vez mais recomendada como um dos componentes importantes para aqueles que desejam uma melhor qualidade de vida, sendo a musculação uma forma de exercitar-se na busca da promoção da saúde e prevenção de doenças ${ }^{3}$. O Conselho Federal de Odontologia (CFO), em 2015, criou uma nova especialidade denominada de Odontologia do Esporte, com a função de estudar, prevenir e tratar as lesões que afetam o sistema estomatognático, bem como manter a saúde bucal de atletas ${ }^{4}$.

Dentre as causas para o desenvolvimento da atrição e abfração destacase o apertamento dentário que às vezes pode estar presente durante a prática de alguns exercícios físicos ${ }^{5}$. Sendo que este apertamento é um ato inconsciente exacerbado, dentre outras situações, por esforços que envolvam forças musculares de contração ${ }^{6}$. Atletas profissionais e praticantes de esportes não profissionais, ingerem bastante bebidas isotônicas durante suas atividades ${ }^{7}$, podendo estas serem consideradas potencialmente erosivas aos tecidos dentais se consumidas de modo inadequado e com elevada frequência ${ }^{8}$. Além disso, os praticantes de musculação exercem forças constantes em suas atividades físicas, sendo possível que esse hábito de força ocorra durante a escovação dos dentes provocando a abrasão dentária ${ }^{1}$.

Apesar da musculação ser uma atividade física muito praticada, a produção científica relacionando esta modalidade com os desgastes dentários que podem acometer seus praticantes não foi identificada na literatura. Desta forma, o presente estudo teve como objetivo analisar a frequência de desgastes dentários em praticantes de exercícios físicos de musculação do município de Caicó - RN, Brasil e avaliar o conhecimento dos mesmos sobre estes desgastes, possibilitando obter subsídios para implantação de ações educativas e preventivas por parte dos cirurgiões-dentistas e educadores físicos de academia a respeito dessas manifestações nos elementos dentários durante a prática de exercícios físicos anaeróbicos.

\section{MATERIAL E MÉTODO}

O presente estudo foi do tipo transversal, com abordagem observacional, exploratória, descritiva e analítica. Foi realizado com 260 praticantes de exercícios físicos de musculação de 5 academias da cidade de Caicó - RN, Brasil. Para chegar a esta amostra, baseou-se na população de 1280 sujeitos (total de praticantes de musculação das academias), bem como considerou-se a proporção de desgastes dentários de $30 \%$, fixando a probabilidade de erro tipo I em $5 \%$ e a probabilidade de erro tipo II em 20\%.

Para a escolha das academias foram incluídas aquelas cadastradas no Conselho Federal de Educação Física (CONFEF) e que ofereciam exercícios físicos de musculação. A amostra foi composta por homens e mulheres entre 18 a 50 anos de idade, que praticavam atividade física de musculação há pelo menos um ano e totalmente dentados ou parcialmente desdentados com contenção cêntrica. Os participantes assinaram 0 Termo de Consentimento Livre e Esclarecido, aprovado pelo Comitê de Ética em Pesquisa da Universidade do Estado do Rio Grande do Norte sob parecer de no 2.032.985.

Para a coleta de dados foi utilizado um questionário com perguntas objetivas e subjetivas, baseado no estudo de Alves et al. ${ }^{9}$. As informações avaliadas incluíam: caracterização da população de estudo (idade, sexo, academia que pratica a atividade de musculação); questões sobre a prática dos exercícios de musculação (há quanto tempo pratica musculação, quantos dias na semana, utilização de quantos aparelhos da academia); perguntas sobre as sensações e costumes durante e após os exercícios de musculação (dores nos músculos da face e/ou dores nos dentes, hábitos de apertar ou ranger os dentes) e questões quanto ao consumo de bebidas antes e depois dos exercícios (água, energéticos, isotônicos).

Também foram abordadas questões sobre condições sistêmicas (refluxo gastroesofágico, regurgitação, sensação de boca seca); hábitos parafuncionais (mascar chiclete, roer unhas, colocar objetos entre os dentes, cortar linha com os dentes); ingestão de alimentos e bebidas ácidas; tipo de escova e uso de dentifrício abrasivo, além de questões sobre o conhecimento dos desgastes dentários pelos praticantes de musculação (capacidade de reconhecer e onde encontrou a informação). Após a aplicação dos questionários, todos os participantes foram submetidos a um exame clínico intraoral dos dentes das arcadas superior e inferior, sendo dois pesquisadores previamente calibrados para diagnosticar os desgastes dentários e para esta análise foram utilizados Equipamentos de Proteção Individual (EPI), tais como espátulas de madeira e luvas de procedimento de látex, ambas descartáveis. Os dados foram coletados em sala reservada dentro da academia, ocasião na qual os 
participantes foram devidamente informados sobre a pesquisa, seus objetivos e benefícios.

Foi realizada análise descritiva dos dados, com apresentação de frequências absolutas e percentuais dos dados qualitativos e medidas de tendência central e variabilidade para dados quantitativos. Para verificar se houve diferença significativa entre as médias de idade quanto a presença de desgaste dentário, foi utilizado teste t de Student. No que concerne a associação entre as variáveis dependentes (presença e tipo de desgaste) com as variáveis independentes foi utilizado o teste do Quiquadrado de Pearson. A correlação de Pearson foi utilizada entre as variáveis: número de desgaste e idade. $\mathrm{Na}$ análise, foi utilizado o programa SPSS 20.0, sendo considerado nível de significância de $5 \%$.

RESULTADOS

A média de idade dos praticantes de musculação foi de 29,9 anos com desvio padrão de 8,5 , sendo a maioria do sexo feminino (151; $58,1 \%$ ). A presença de pelo menos um tipo de desgaste foi observada em 205 (78,9\%) participantes. No que concerne ao tipo de desgaste que cada participante apresentava, $171(65,8 \%)$ tinham apenas um tipo de desgaste, $32(12,3 \%)$ dois tipos e $2(0,8 \%)$ três tipos. Apesar de 205 praticantes de musculação apresentarem desgastes dentários, parte destes continham mais de um tipo, que resultou em um total maior de tipos de desgastes apresentados $(n=241)$, conforme verificado na Figura 1.

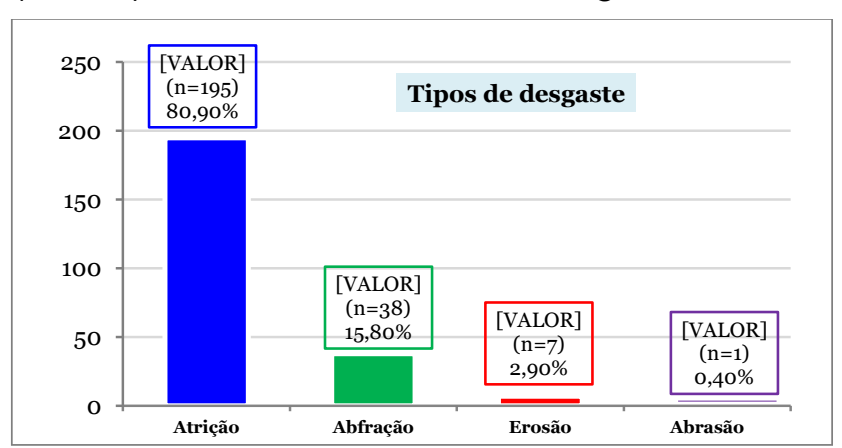

Figura 1: Proporção de desgastes dentários apresentados pelos praticantes de musculação (atrição, abfração, erosão e abrasão). Caicó - RN, Brasil 2019.

A atrição e a abfração foram mais prevalentes no sexo feminino, sendo 107 $(70,9 \%)$ mulheres com atrição e $26(17,2 \%)$ com abfração. Dentre os praticantes de musculação que frequentavam academia até 4 dias por semana, 71 (78,9\%) apresentavam atrição e 19 $(21,1 \%)$ abfração.

A maioria dos participantes da pesquisa era praticante de musculação há mais de 3 anos $(n=140 ; 53,8 \%)$ e a maioria destes utilizavam até 08 aparelhos da academia por dia $(n=228$; $87,7 \%$ ). Analisando as sensações e costumes durante e após os exercícios de musculação, 9 $(3,5 \%)$ apresentavam dores nos músculos da face e/ou dores nos dentes durante os exercícios e $4(1,5 \%)$ após os exercícios; 83 $(31,9 \%)$ apertavam os dentes durante os exercícios e 19 (7,3\%) rangiam os dentes durante a prática de musculação. No presente estudo também foram observados outros possíveis fatores relacionados com a presença de desgastes dentários nos praticantes de musculação, conforme consta na Tabela 1.

Tabela 1. Frequência dos possíveis fatores relacionados com os desgastes dentários nos praticantes de musculação. Caicó - RN, Brasil 2019

\begin{tabular}{lcc}
\multicolumn{1}{c}{ Fatores } & n & $\%$ \\
\hline Consumo de bebidas antes dos exercícios & 151 & 58,1 \\
\hline Água & 37 & 14,2 \\
\hline Café & 08 & 3,1 \\
\hline Energético & 157 & 60,4 \\
\hline Consumo de bebidas após os exercícios & 23 & 8,8 \\
\hline Água & 52 & \\
\hline Suco & 47 & $\mathbf{2 0}$ \\
\hline Alterações gastrointestinais & 82 & 31,5 \\
\hline Regurgitaçã & & \\
\hline Refluxo gastroesofágico & 174 & 66,9 \\
\hline Sensação de boca seca & 165 & 63,5 \\
\hline Consumo diário de alimentos/bebidas erosivas & & \\
\hline Alimentos ácidos & 80 & 30,8 \\
\hline Bebidas ácidas & 71 & 27,3 \\
\hline Hábitos parafuncionais & 67 & 25,8 \\
\hline Colocar objeto entre os dentes & 64 & 24,6 \\
\hline Mascar chiclete & & \\
\hline Cortar linha com os dentes & 67 & 25,8 \\
\hline Roer unhas & & \\
\hline Instrumentos de higiene oral & & \\
\hline Escova de cerdas duras e dentifrício abrasivo & & \\
\hline
\end{tabular}

O teste de Qui-quadrado de Pearson foi aplicado para verificar a associação entre as variáveis independentes e os seguintes tipos de desgastes: atrição e abfração. Já para os desgastes do tipo erosão e abrasão, o teste não foi aplicado devido a pequena quantidade de ambas as lesões. Sendo assim, fatores como consumo de alimentos, bebidas ácidas e fatores gastrointestinais não foram relacionados com a erosão pela pouca frequência da lesão. Os hábitos parafuncionais como roer unhas e colocar objetos entre os dentes, bem como o uso de escova de cerdas duras e dentifrício abrasivo não foram relacionados com a abrasão, também pela pouca frequência. Hábitos de ranger ou apertar os dentes durante os exercícios e sentir dores nos músculos da face e/ou dores nos dentes durante e depois das atividades não tiveram associação significativa com atrição e abfração $(p>0,05)$.

Não foi observada associação significativa entre o sexo e a presença dessas lesões. A média de idade dos indivíduos foi maior e a diferença estatisticamente significativa entre os que apresentavam atrição e abfração, conforme verificado na Tabela 2 . Além disso, a quantidade de desgastes foi correlacionada positivamente com a idade $(p<0,001), r^{2}=0,39$, ou seja, quanto maior a idade, maior a quantidade de desgastes. Houve também associação estatisticamente significativa 
$(p=0,031)$ entre ter abfração e frequentar academia até 4 vezes na semana, enquanto que para atrição essa associação não foi verificada $(p=0,292)$.

Tabela 2. Diferença entre as médias de idade entre os indivíduos com e sem lesão de atrição e abfração. Caicó - RN, Brasil 2019.

\begin{tabular}{lcc} 
Tipo de desgaste & \multicolumn{1}{c}{ Mdade } & \\
& $\begin{array}{c}\text { Média } \\
\text { (desvio padrão) }\end{array}$ & $p$ \\
Atrição & $30,88(8,57)$ & \\
Sim & $26,94(7,52)$ & 0,001 \\
\hline Não & & \\
Abfração & $36,37(8,82)$ & $<0,001$ \\
\hline Sim & $28,78(7,92)$ & \\
\hline Não & & \\
\hline
\end{tabular}

No que se refere ao conhecimento dos praticantes de musculação sobre os desgastes dentários, observou-se que 91,9\% não tinham conhecimento sobre os desgastes. Dentre estes, 97,7\% não conheciam atrição e abfração e $95,8 \%$ não conheciam erosão e abrasão. Os participantes que afirmaram ter conhecimento sobre os desgastes citaram a abrasão e a erosão como as lesões mais conhecidas, sendo o cirurgião-dentista o profissional mais citado como fonte de informação sobre os quatro tipos de desgastes dentários.

DISCUSSÃO

Estudos vêm sendo desenvolvidos buscando definir os fatores etiológicos para o surgimento da atrição, abfração, erosão e abrasão. Estes desgastes dentários possuem causas multifatoriais, sendo atribuído diversos fatores etiológicos para a formação destas lesões $^{10,11}$. No estudo de Silva e Bonis ${ }^{5}$, os autores observaram que dentre os fatores odontológicos mais comuns e de maior relevância que podem causar deficiência no rendimento dos atletas estão o apertamento dos dentes e a dor na articulação temporomandibular.

Diversos motivos levam as pessoas à procurarem a musculação como forma de exercitarem-se, sejam estes motivos estéticos, prevenção à saúde, reabilitação, combate ao sedentarismo e recomendações médicas. Como qualquer outra prática de exercícios físicos, a musculação possui seus riscos quando praticada de maneira incorreta, uma vez que seus praticantes podem sofrer ou agravar algum tipo de lesão, seja ela óssea, muscular, ligamentar ou tendínea ${ }^{3}$.

De acordo com o estudo de Soares e Silva ${ }^{3}$ os praticantes de musculação não citaram lesões nos órgãos do sistema estomatognático, podendo inferir que essa associação entre musculação e lesões nos órgãos do sistema estomatognático não foi percebida por eles. Em contrapartida, Cordeiro et al. $^{4}$ avaliaram a relação entre a prática esportiva do surf com os tipos de lesões bucofaciais, sendo verificado que as fraturas dentárias foram as lesões mais prevalentes entre os surfistas.

Hábitos de apertar os dentes ou ranger durante a prática de exercícios físicos de musculação não tiveram associação significativa com a presença de lesões, dados condizentes ao estudo de Oliveira et al. ${ }^{10}$, em que não houve relação entre os hábitos parafuncionais, alimentares e de higiene com a presença de lesões. Neste estudo não houve associação significativa entre sexo e presença de lesões, resultado este concordante com os relatos de Oliveira et al. ${ }^{10}$, Sousa et al. ${ }^{11}$ e Pegoraro et al. ${ }^{12}$ que também não encontraram associação entre o sexo e a ocorrência de lesões. No entanto, no presente estudo, a atrição e a abfração foram mais prevalentes no sexo feminino, sugerindo que alguns fatores desencadeantes das lesões podem estar relacionados às mulheres, tais como estresse, ansiedade e fatores hormonais que podem levar a mudanças na alimentação, desenvolvimento de hábitos e falta de cuidado com os elementos dentários.

Para alguns autores ${ }^{12,13}$, a idade não é um fator que contribui para o desenvolvimento das lesões dentárias de desgaste. Diferentemente, em outros estudos ${ }^{10,11,14}$, os autores concluíram que a presença de desgastes dentários estava associada com o avanço da idade dos pacientes, dados que coincidem com os resultados do presente estudo, no qual observou-se que lesões de atrição e abfração foram mais prevalentes em indivíduos com idade mais avançada. Isso pode ser explicado pela exposição dos dentes de pessoas mais velhas aos fatores etiológicos de origem fisiológica e/ou patológica no decorrer do tempo. Adicionalmente, observou-se que quanto maior a idade, maior a quantidade de desgastes, resultado que diverge do trabalho de Molena et al. ${ }^{13}$ no qual $o$ número de dentes acometidos não apresentou relação com a idade.

Soares e Silva ${ }^{3}$ relataram a presença de algum tipo de lesão no corpo proveniente dos exercícios físicos de musculação entre praticantes que frequentavam a academia de 4 a 5 vezes por semana, semelhante ao presente estudo, em que a abfração foi mais prevalente naqueles que frequentavam academia até 4 dias por semana. Assim, é perfeitamente plausível que entre os praticantes de musculação, o tempo de prática, a frequência de treinamento e a quantidade de aparelhos utilizados na academia possam interferir 
diretamente no aparecimento dos desgastes dentários, bem como de lesões corporais.

No que diz respeito a autoavaliação dos praticantes de musculação sobre os desgastes, um valor expressivo não tinha conhecimento sobre eles. Algumas causas podem ser sugestivas para essa falta de conhecimento, como a deficiência na comunicação entre profissional e paciente, como destaca o estudo de Ribeiro et al. ${ }^{15}$, no qual observou-se que os cirurgiões-dentistas, apesar de demonstrarem conhecimento sobre 0 tratamento destes desgastes, exibiram resultados mais divergentes com relação ao diagnóstico das lesões. Dessa forma, a dificuldade no diagnóstico apresentada pelos profissionais pode refletir, consequentemente, na ausência do diálogo com o paciente sobre os desgastes dentários. Outro fator relevante é a desigualdade de oferta dos serviços de saúde associada a baixa condição socioeconômica, como apontado no estudo de Peres et al. ${ }^{16} \mathrm{em}$ que os menos escolarizados têm maiores dificuldades de acesso aos serviços odontológicos.

Gonçalves e Deusdará ${ }^{17}$ ressaltam em seu estudo que devido a elevada frequência dos desgastes dentários, faz-se necessário um maior conhecimento das etiologias para um adequado diagnóstico e prevenção. A erosão e abrasão foram as lesões menos frequentes nos indivíduos do presente estudo, sugere-se que isso pode ter acontecido devido tais lesões serem as mais conhecidas por eles e de certa forma consigam preveni-las, contudo, novos estudos devem ser feitos para investigar essa relação.

Partindo destas premissas, verifica-se a importância da atuação da Odontologia no cotidiano da prática de esportes, como em clubes, academias e em escolas, visando contribuir com a saúde bucal dos atletas e praticantes de exercícios físicos como um todo ${ }^{18}$, uma vez que a cavidade bucal pode ser susceptível a lesões decorrentes de hábitos nesses grupos.

Quanto as limitações desta pesquisa, destaca-se a inexistência de estudos sobre o tema abordado, o que dificultou comparações entre trabalhos de metodologias semelhantes, como também a não inserção de outros fatores etiológicos para verificar a presença de desgastes dentários nos praticantes de musculação. Assim, como os desgastes dentários apresentam causas multifatoriais, novos estudos devem ser realizados a fim de esclarecer outros fatores etiológicos envolvidos no aparecimento destas lesões nos praticantes de musculação.

CONCLUSÃO

A presença de pelo menos um tipo de desgaste ocorreu em 205 praticantes de musculação, sendo a atrição e a abfração os desgastes mais frequentes, seguidos de erosão e abrasão. O avanço da idade é um fator importante para o aparecimento da atrição e da abfração e quanto maior a idade, maior a quantidade de desgastes dentários. 0 conhecimento dos praticantes de musculação sobre tais desgates foi escasso, sendo a atrição e a abfração as mais desconhecidas, podendo implicar em deficiências na prevenção, uma vez que o presente público se mostrou susceptível ao desenvolvimento destas lesões.

A atuação do cirurgião-dentista juntamente com uma equipe multiprofissional, dentre estes, o educador físico, através da implantação de medidas educativas e preventivas, pode melhorar o rendimento dos praticantes de musculação em suas atividades, visto que é cada vez mais evidente a relação entre a Odontologia e as práticas esportivas.

\section{REFERÊNCIAS}

1. Amaral SM, Abad EC, Maia KD, Weyne S, Oliveira MPRPB, Tunãs ITC. Lesões não cariosas: 0 desafio do diagnóstico multidisciplinar. Arq Int Otorrinolaringol. 2012; 16(1):96-102.

2. Brentegani LG, Lacerda SA, Campos AA. Alterações regressivas dos dentes: abfração; abrasão, atrição e erosão. Rev Bras Teleodonto. 2006;1(1):11-7.

3. Soares EMS, Silva KMA. Prevalência de lesões em praticantes de musculação em academias do município de Morada Nova/CE. BIUS. 2018; 9(1):67-78.

4. Cordeiro JBF, Forte LB, Neri JR, Santos SE, Gomes FA, Lima DLF. Fatores etiológicos e prevalência de lesões bucofaciais em surfistas de Fortaleza. Rev Bras Ciênc Esporte. 2018;42:e2002.

5. Silva AMG, Bonis R. Odontologia desportiva: a influência da saúde bucal na performance dos atletas da Vila Olímpica de Manaus. BIUS. 2018; 9(1):79-91.

6. Lemos LFC, Oliveira RS. Odontologia desportiva: uma breve revisão sobre essa nova tendência no esporte. Rev Digital. 2007; 12(113).

7. Zandim DL, Gilio $C$, Rossa Júnior $C$, Sampaio JEC. Influência de bebidas isotônicas na remoção de smear layer de superfícies radiculares após raspagem. Estudo in vitro. Rev Odontol UNESP. 2008; 37(3):267-73. 
8. Cavalcanti AL, Xavier AFC, Souto RQ, Oliveira MC, Santos JA, Vieira FF. Avaliação in vitro do potencial erosivo de bebidas isotônicas. Rev Bras Med Esporte. 2010; 16(6):455-58.

9. Alves MSC, Lucena SC, Araujo SG, Carvalho ALA. Diagnóstico clínico e protocolo de tratamento do desgaste dental não fisiológico na sociedade contemporânea. Odontol ClínCient. 2012;11(3): 247-51.

10. Oliveira ACS, Damascena NP, Souza CS. Análise clínica de pacientes portadores de lesões cervicais não cariosas e sua relação com hábitos. Rev Sul-Bras Odontol. 2010; 7(2):182-92.

11. Sousa AMT, Prado RA, Filho AAC. Fatores de risco oclusais e sua influência na etiologia das lesões cervicais não-cariosas. Revista Dentística online. 2012; 23:19-25.

12. Pegoraro LF, Scolaro JM, Conti PC, Telles D, Pegoraro TA. Noncarious cervical lesions in adults: prevalence and occlusal aspects. J Am Dent Assoc. 2005;136(12):1694-700.

13. Molena CCL, Rapoport A, Rezende CP, Queiroz CM, Denardin OVP. Relação entre lesões cervicais não cariosas e hábitos. Rev Bras Cir Cabeça Pescoço. 2008;37(4): 20611.

14. Santos TS, Moura AD, Piva MR, Carvalho LPM, Filho PRSM. Relação dos desgastes dentários com as disfunções temporomandibulares. Rev cir traumatol buco-maxilo-facial. 2009;9(2):7380.

15. Ribeiro VSCR, Almeida EL, Leal IC, Bizerril DO, Saintrain MVL, Ferreira RGLA et al. Diagnóstico e tratamento de lesões não cariosas: a visão do cirurgião-dentista do sistema público de saúde. J Health Biol Sci. 2019;7(2):204-10.

16. Peres MA, Iser BPM, Boing AF, Yokota RTC, Malta DC, Peres KG. Desigualdades no acesso e na utilização de serviços odontológicos no Brasil: análise do Sistema de Vigilância de fatores de risco e proteção para doenças crônicas por inquérito telefônico (VIGITEL 2009). Cad Saúde Pública. 2012;28(Sup):S90100.

17. Gonçalves PE, Deusdará ST. Lesões cervicais não cariosas na prática odontológica atual: diagnóstico e prevenção. Rev Ciênc Méd. $2011 ; 20(5-6): 145-52$.

18. Pastore GU, Moreira M, Bastos R, Galotti M, Leonardi MFP. Odontologia do Esporte- uma proposta inovadora. Rev Bras Med Esporte. 2017;23(2):147-51.

\section{CONFLITO DE INTERESSES}

Os autores declaram não haver conflitos de interesse

\section{AUTOR PARA CORRESPONDÊNCIA}

\author{
Mariana Silva de Bessa \\ Universidade do Estado do Rio Grande do Norte-UERN, \\ Av. Rio Branco, 725, Centro, \\ 59.300-000 Caicó - RN, Brasil \\ tel. (84) 99960-9993 \\ E-mail:melrybessa@hotmail.com
}

Submetido em 14/08/2020

Aceito em 04/10/2020 